This is a self-archived - parallel published version of this article in the publication archive of the University of Vaasa. It might differ from the original.

\title{
Operative factors affecting energy balancing and speed of equalization in battery storage system
}

Author(s): Palizban, Omid; Kauhaniemi, Kimmo

Title: Operative factors affecting energy balancing and speed of equalization in battery storage system

Year: $\quad 2019$

Version: Accepted manuscript

Copyright (C)2019 IEEE. Personal use of this material is permitted. Permission from IEEE must be obtained for all other uses, in any current or future media, including reprinting/republishing this material for advertising or promotional purposes, creating new collective works, for resale or redistribution to servers or lists, or reuse of any copyrighted component of this work in other works.

\section{Please cite the original version:}

Palizban, O., \& Kauhaniemi, K., (2019). Operative factors affecting energy balancing and speed of equalization in battery storage system. In: 2018 IEEE Electrical Power and Energy Conference (EPEC), Toronto, ON. IEEE. https://doi.org/10.1109/EPEC.2018.8598279 


\section{Operative Factors Affecting Energy Balancing and Speed of Equalization in Battery Storage System}

\author{
Omid Palizban \\ School of Technology and Innovations \\ University of Vaasa \\ Vaasa, Finland \\ Omid.Palizban@uva.fi
}

\author{
Kimmo Kauhaniemi \\ School of Technology and Innovations \\ University of Vaasa \\ Vaasa, Finland \\ Kimmo.Kauhaniemi@uva.fi
}

\begin{abstract}
Energy storage systems play a significant role in power management systems and control of the modern grid. One of the most challenging issues is controlling storage units in distributed form. This paper presents a possible means of controlling Energy Storage Systems (ESS) through a decentralized approach. Moreover, the balancing and equalization of stored energy in different storage units presents other challenges in such systems; to deal with this, the paper discuss here the factors that affect energy balancing and the speed of the energy balance convergence. A ProportionalIntegral (PI) controller is used in the upper control level to generate an accurate reference value for the State of Charge (SoC), and a modified droop control is employed on the lower control level to equalize the energy on the basis of the SoC. To evaluate the control algorithm and to investigate the factors that affect the speed of equalization, this paper considers the result of a case study with three battery storage units.
\end{abstract}

Keywords - Battery, Droop Control, Energy Balancing, Energy Storage System, Hierarchical Control

\section{INTRODUCTION}

Power management and stability assurance are critical in modern grids because of the variables involved on the generation and demand sides. Using storage to absorb and inject energy as needed often serves as the best solution to manage this issue. Managing power balance and stability is a challenging task in energy storage systems, and a hierarchical control system is needed to achieve optimum performance. Hierarchical control has been discussed comprehensively for all kinds of inverter-based sources in [1]; it consists of four different levels, for processing (inner control loop), sensing and adjusting (primary level), monitoring and supervising (secondary level), and maintenance and optimization (tertiary level). Electrochemical storage technology (battery storage) is the most popular technique on account of its broad capacity range, fast and flexible dynamic response, and high efficiency [2]. A simplified Battery Energy Storage System (BESS) is shown in Figure 1. A typical BESS consists of a battery pack, a Battery Management System (BMS), a monitoring and control unit-known as a Power Control System (PCS) - protective circuitry, filters, and a grid connection. Hierarchical control is the part of the monitoring and control unit that coordinates both functional and optional operation of a BESS. Optimal control for a BESS should involve the two following aspects: First, the BMS should ensure energy balancing for each cell of the battery storage system; second, the PCS should ensure overall equalization of the SoC in the battery storage units [3][4]. This can be implemented by estimating the $\mathrm{SoC}$ as well as measuring voltage, frequency and any fluctuation in the electricity provided by resources and load variation on demand side. There are several key challenges that can affect BESS operation and a number of technical possibilities that allow each control level of the system to provide optimum power management. Vandoorn et al. [5] present a complete review of primary techniques based on local measurements only. Comprehensive research into hierarchical control and the secondary control level is presented by Guerrero et al. in [6]. Based on their investigation, secondary control levels can be classified into two types: centralized and decentralized; the challenges of the control level, with solutions, are presented in [7]. Since the level of stored energy is different in each battery unit, a major challenge in control strategies is to balance the energy in the system. The fundamental control (primary level) maintains the power balance in output and bus frequency, adjusts the voltage, and determines the safe reference operation by taking account of the SoC of each unit. The upper control level (Secondary control) optimizes the distribution power and regulates frequency and voltage to synchronize with the upper unit (microgrid or main grid). The reference values of the functional measurement, such as voltage $\left(V_{r e f}\right)$ and frequency $\left(f_{r e f}\right)$, are obtained on this level; recently, several methods have been developed to generate these set point values on the secondary level [8][9]. Moreover, the total energy stored in the different units and the $\mathrm{SoC}$ of each unit are determined on the upper control level. The main research question in this area concerns possible techniques for sharing power between storage units based on the available energy in each unit and the factors that increase the speed of convergence. To answer the above research questions, the objective of this paper is to investigate different methods of the energy balancing and to propose a distribution technique to equalize the SoC of the storage units. Furthermore, factors for utilizing rapid convergence between the energy level of each unit and output power of them are discussed.

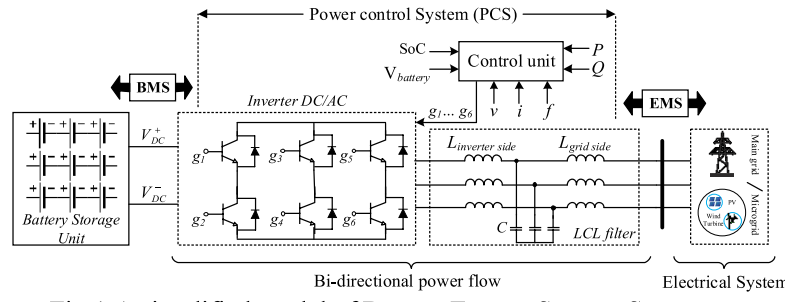

Fig.1 A simplified model of Battery Energy Storage System

The paper is organized as follows: the principles of SoC coordination are presented in Section 2. Section 3 discusses 
the equalization of energy for BESS in detail. In Section 4, a simulation study is presented to evaluate the performance of the energy balancing and speed regulation. Finally, the paper is concluded in Section 5.

\section{PRINCIPLES OF SOC COORDINATION IN BESS}

In the modern grid, to support most challenging tasks regarding variation and fluctuation of power generationsuch as frequency regulation, voltage support, and power management - the storage units can be utilized. In this regard, the stored energy or SoC must be sufficient during operation, and there are several methods to implement an optimal control strategy for equalizing the values [10]. The stored energy in the storage units is generally calculated based on the ratio of available capacity of energy $\left(Q_{t}\right)$ to the maximum capacity $\left(Q_{\max }\right)$, the result being a value between 0 (full discharge) and 1 (full charge) $(0 \leq \mathrm{SoC} \leq 1)$. Indeed, of the several methods used to calculate these values [11], the coulomb-counting technique demonstrated below is most popular, due to its simplicity and low cost [12].

$$
S o C=S o C_{t=0}-\int \frac{i d t}{C_{i}}
$$

Where $\mathrm{C}_{i}$ is capacity of the storage unit $(i), i$ is output current of the unit based on time. Estimating the level of energy is essential in operating storage units because of the effect on the system performance. The decision to charge or discharge progress is made based on the measurement and reference values that are generated by the control unit. There are several different control methods algorithms for defining the reference value. Therefore, coordinating the $\mathrm{SoC}$ in BESS and the principles of the control strategy are discussed in the next section.

\section{A. Battery inverter controls}

Control of the power electronics inverter for all kind of inverter-based sources, including batteries, has been intensively studied in recent years [10]. Hierarchical control consist of three different levels: primary, secondary, and tertiary [13][14]. An overview of the control system interconnection is shown in figure 2. The control algorithm for SoC is part of the primary and secondary control levels, so that the reference value of the SoC is generated on the second level and the sharing of power based on the available energy is the responsibility of the primary control.

Droop control and its variants are the most common primary methods for integrating several voltage inverter based sources in storage units [15][16]. Droop control linearly define new reference value for frequency (in AC) and voltage (in DC) when there is variation in output power [17]. Equation 2 describes the standard droop control used to control an $\mathrm{AC}$ system; Equation 3 is the equivalent for a DC system.

For AC system:

$$
f=f^{r e f}-G_{p(s)}\left(P-P^{r e f}\right)
$$

For DC system:

$$
V=V^{r e f}-G_{p(s)}\left(P-P^{r e f}\right)
$$

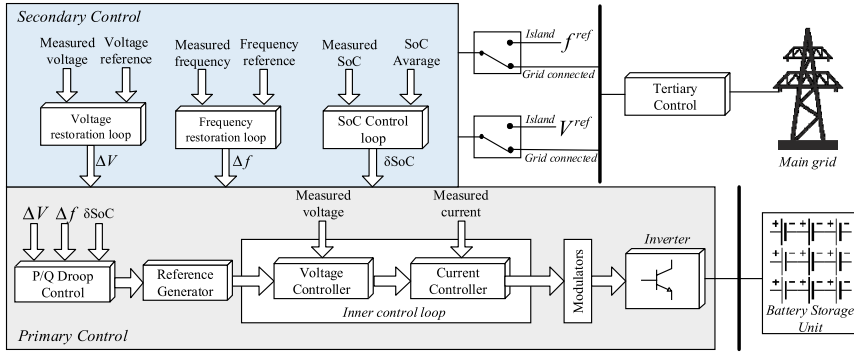

Fig.2 Hierarchical control for Battery Storage unit

During the charging and discharging processes, the SoC of each storage unit should be balanced and the power output or injected should be based on the SoC of the unit. Since the sharing of power between different storage units is based on power capacities, rather than on energy levels, some malfunction of the droop control can occur; there are also several general limitations on facilitating power sharing between the units [18][19]. It is thus necessary to modify the conventional droop control in order to overcome these limitations.

\section{B. Management Systems in BESS}

The monitoring and control unit in a BESS is responsible for the interface that coordinates the operation of the system. Management can be carried out by receiving values from the internal measurements, such as SoC, and external measurements, such as the voltage at the PCC. As mentioned earlier, the control units consist of the two different levels: BMS and PCS. The BMS balances the stored energy in each cell of the battery, and cannot be used to equalize the energy of individual batteries in a storage unit. Hence, this control section is not capable to implement a management system for BESS, and cannot make an interface between the storage units and distributed generation systems (e.g Microgrid). To deal with the leakage of energy, and to equalize $\mathrm{SoC}$ in a storage unit, PCS should be implemented in the BESS [17]. A BMS may be passive or active. Passive cell equalization, also called as dissipative charge balancing, operates using approaches that inject energy based on the current energy level. For instance, a cell with a higher rate of SoC will inject more energy into the system than a cell with a lower value.

Hence, after a short period, the levels of SoC in all cells become similar. Active cell balancing is a better method when compared to the passive method since it reduces power losses. In the active technique, an energy interface is used between different cells to transfer energy from a cell with higher SoC to a cell with a lower SoC. Unlike the passive method, which is utilized for lead-acid and nickelbased technologies, the active method does not depend on characteristics of the cell and can be implemented in all battery technologies. There are several methods of utilizing the active method to balance energy in batteries; however, since this paper focuses on PCS, these are not discussed here. A comprehensive and detailed classification of active methods can be found in [12]. 


\section{ENERGY BALANCING FOR STORAGE UNITS}

As mentioned before, droop control is the most popular technology for implementing primary control. However, the traditional version of droop control shares power between different units based on the rated power, rather than on the energy available in each storage unit. This type of power sharing can lead to some problems in the control system, if storage units with lower energy levels run out of energy earlier than the other units; it leads to causing an instability of frequency in discharging period. Moreover, when the storage units with the highest energy levels become full, and drawing of energy ceases to be possible, even when the power demand is lower that the generated power-which would lead to renewable power being wasted due to unavailable storage capacity. To address this challenge, a modified droop control is required for the primary level, based on the SoC of the storage units. The energy should be balanced during both the charging and discharging process, and their output or injected power should be based on the SoC of the same storage unit.

\section{A. Modified droop control in BESS}

In modern grids, due to the use of inverter-based sources, the lack of the inertia is a significant challenge. Active and reactive power, frequency, and voltage are thus managed using primary control (the droop method). For instance, in AC networks, the frequency of the system is used to sense the capacity of the network. If the measured value $(f)$ is close to the maximum value $\left(f_{\max }\right)$, it is an indication that the power generated in the system is greater than the demand, and the BESS should therefore absorb the excess power, taking the level of stored energy (SoC) in each unit account. On the other hand, during a shortage of power (e.g., caused by an increase in the load), the measured frequency $(f)$ is close to the minimum value $\left(f_{\min }\right)$ and the BESS should start to discharge, injecting power into the system. Taking into consideration the brief explanation of the principle of control in the droop method, it could be concluded that the measured values - such as frequency, voltage, and output power-are not constant during operation, and may also differ point by point due to line impedance (measured value of voltage). Hence, the best way to optimize the conventional droop method is to modify the value of the droop coefficient $\left(G_{p(s)}\right.$ in Eqs. 2 and 3). In a DC system, there are usually not a large number of voltage drops in the power cable (e.g., less than $0.13 \mathrm{~V}$ for a $1 \mathrm{~km}$ transmission line supported by $2 \mathrm{~kW}$ active power) [17]. Moreover, in an AC system, the frequency is the same everywhere and the difference between the measured value and the reference value ( $\Delta f)$ is constant. Hence, Eq. 2 and Eq. 3 can expressed as follows:

For AC system:

$$
f_{\text {unit }_{1}} \approx f_{\text {unit }_{2}} \approx \ldots \approx f_{\text {unit }_{n}}
$$

For DC system:

$$
V_{\text {unit }_{1}} \approx V_{\text {unit }_{2}} \approx \ldots \approx V_{\text {unit }_{n}}
$$

and the droop control may be expressed as:

$$
P_{1} G_{p(1)}=P_{2} G_{p(2)}=\ldots=P_{n} G_{p(n)}
$$

Based on the equations, it is clear that, to have optimum control, the droop coefficients should be set inversely proportional during the discharge and proportional during the charging period.

Recently, energy balancing and equalization of energy levels has become an interesting topic to research, and several methodologies have been proposed for DC and AC systems, such as that of X. Lu et al. [17] and Morstyn et al. [20]. The principle of equalization in these methods is to multiply the droop coefficient by deviation of SoC $\left(\delta_{\mathrm{SoC}}\right)$ to indicate the level of energy in each storage unit. The value falls between a minimum of 0.01 (discharged) and a maximum of 1 (charged), so as to prevent the droop coefficient from going to infinity. The following equations demonstrate the above explanation in a mathematical way.

For Charging:

$$
G_{p(o l d)}=G_{p(s)} \times \delta_{S o C}
$$

For discharging:

$$
\begin{aligned}
& G_{p(o l d)}=G_{p(s)} / \delta_{S o C} \\
& \text { and } \\
& \delta_{S o C}=\max \left(V-V_{\min } / V_{\max }-V_{\text {min }}, 0.01\right)
\end{aligned}
$$

The risks of these control methods are high because of problems that arise from these limitations and lead to significant problems in the control of the BESS - such as overcharging and over discharging. Hence, it is important to optimize the method so that there is no limitations or risks related to the energy balancing. In this regard, the inverse of the previous methods - employing multiplication or division of the variable - is used; in the new method, the droop coefficient is determined by adding (charging) or subtracting (discharge) the deviation of the available energy level (SoC) to or from the droop coefficient value. With this method, the above limitation (Eq. 9) is no longer relevant, so if the restoration signal goes to zero, the droop coefficient still is equal to the set amount. Hence, the new droop coefficient value $\left(G_{p(n e w)}\right)$ is defined as:

For charging period:

$$
G_{p(\text { new })}=G_{p(s)}-\delta_{S o C}
$$

For discharging period:

$$
G_{p(\text { new })}=G_{p(s)}+\delta_{S o C}
$$

\section{B. Energy-balancing speed adjustment}

In BESS control, an important value for implementing optimized control for the system is the total amount of stored energy in all units. In the second step, the average value is calculated and the $\mathrm{SoC}$ of each unit should be determined. This algorithm is a part of the secondary control level, shown in Figure 3.

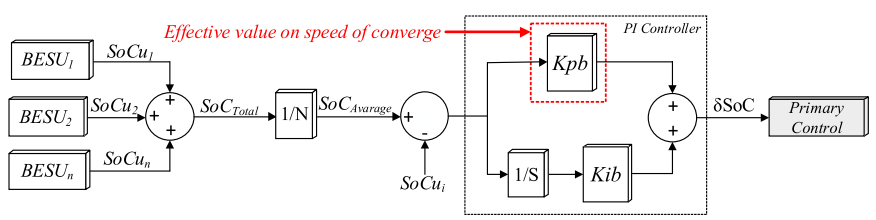

Fig.3 The algorithm of energy balancing in secondary control level 
In this control strategy, the average value of the SoC $\left(S o C_{\text {avarage }}\right)$ is calculated by dividing the total available SoC $\left(S o C_{t o t a l}\right)$ by the number of battery storage units $(\mathrm{N})$. The deviation in each BESU is then obtained by comparing the with the SoC value of the same BESU $\left(S o C u_{i}\right)$. A simple PI controller that is sufficiently fast to avoid full charge or discharge is added, in order to determine the appropriate SoC value. The output of the PI controller is a control signal in every sample period $(\delta S o C)$, which is added to the droop coefficient in primary control. In the modified droop control method, the unit with the lowest energy level provides the least power, compared with the unit with the highest energy level to support the load and system. The energy balancing and power sharing can be adjusted by changing the proportional gain on the secondary level. The PI controller, which is also used to determine the control signal for the energy level of each storage unit, amplifies and integrates the deviation of the energy level so as to remove the error from the system. Thus, the increase is directly proportional to the increase in the speed of the power-sharing adjustment and the reduction in the difference between the energy levels. A flowchart of the control algorithm and speed regulation are shown in Figure 4 and is validated in the next section.

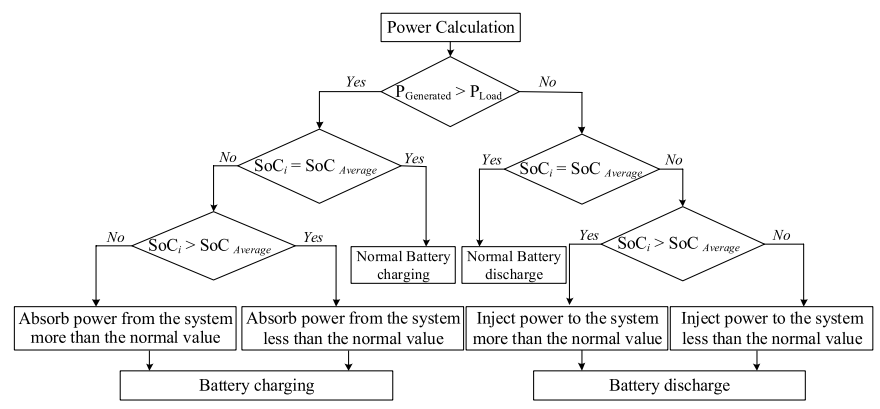

Fig.4 A flowchart of energy regulation in BESS

\section{CASE STUDY}

To evaluate the control methodology and speed adjustment for BESS, the configuration of the studied system is shown in Figure 5; it consists of three storage units (lithium-ion batteries) with distributed generation (a DC voltage source model) connected to an AC bus through a power electronics interface (the capacity of the inverter is $1.5 \mathrm{~kW})$. The available energy in each unit for both periods (charging and discharging) is demonstrated in the figure, with the initial value of the SoC being adjusted in 5\% steps.

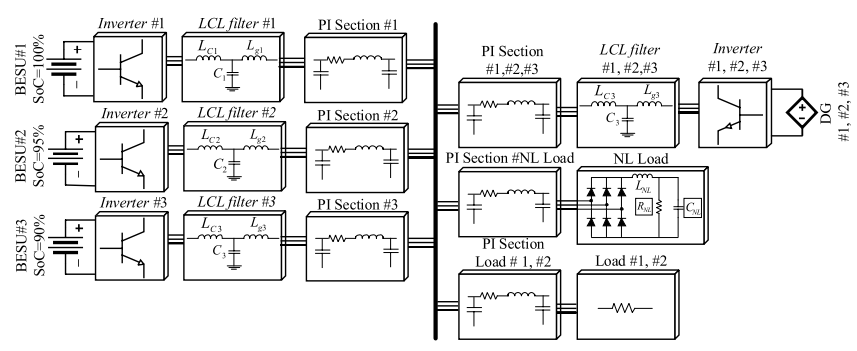

Fig.5 The configuration of the studied system

\begin{tabular}{|l|c|c|}
\hline \multicolumn{3}{|c|}{ Table.1 Simulation parameters } \\
\hline SoC proportional term & $K_{p b}$ & $0.25 \times 10^{3}$ \\
\hline SoC Integral term & $K_{i b}$ & 3 \\
\hline Filter inductance & $L_{c}$ & $L_{g} / 2$ \\
\hline Output impedance & $L_{g}$ & $600 \mathrm{mH}$ \\
\hline Filter capacitance & $C_{b}$ & $100 \mu f$ \\
\hline Reference value of frequency & $f_{r e f}$ & $50 \mathrm{~Hz}$ \\
\hline Reference value of voltage & $V_{r e f}$ & $400 \mathrm{~V}$ \\
\hline
\end{tabular}

To model the distances and distribution lines, a nominal pi-section model is used with different intervals; the AC bus supplies linear and nonlinear loads in a balanced three-phase system. The equalization of the energy levels for each storage unit is illustrated in Figure 6 (charging periods) and Figure 7 (discharging periods), and it can be seen that the storage units with different energy levels approach each other gradually. $\left(\Delta \mathrm{SoC}=\mathrm{SoC}_{\text {unit3 }}-\mathrm{SoC}_{\text {unit1 }}\right.$, due to highest rate of difference is between these units, $\approx 10 \%$ )

To evaluate the role of the proportional gain of the secondary control level in the SoC part, four different values of the gain are considered in this evaluation, the result of which is shown in Figure 8. As shown in the figure, as the value of proportional gain increases, the difference in the stored energy reduces faster, and the equalization of energy at highest rate of $K_{p b}$ takes almost half the time of the equalization with the lowest gain value. For instance, the difference at $\mathrm{t}=10 \mathrm{~s}$ is less than $2 \%$, whereas the value for lowest rate of $K_{p b}$ at the same time is more than $4 \%$. The energy balancing is complete at $\mathrm{t}=20 \mathrm{~s}$ for $K_{p b}=350$ and $\mathrm{t}$ $=40 \mathrm{~s}$ when the value is at its lowest $\left(K_{p b}=200\right)$. Moreover, to analyze the effect of the changes on power management, Figure 9 illustrates the difference between the power injected into the system for the same units during energy equalization $\left(\Delta \mathrm{P}=\mathrm{P}_{\text {unit3 }}-\mathrm{P}_{\text {unit1 }}\right)$. As demonstrated in the figure, the difference between the first and third storage units also gradually approaches zero, and the speed of equalization becomes faster by increasing the proportional gain. Power sharing management is exactly same as the energy balancing, and the time for equalization is twice longer for lowest rate than at the highest rate of $K_{p b}$. For instance, the power difference is close to $40 \mathrm{~W}$ when $\mathrm{t}=20 \mathrm{~s}$; at the same time, for $K_{p b}=350$, the difference value is less than $20 \mathrm{~W}$. Despite employing the method and increasing the speed of energy balancing and power sharing, the increase in the proportional gain leads to overshooting of the output power at the starting point. In Figure 10, the first 10 seconds of the power sharing is shown; it indicates that the overshooting of $\Delta \mathrm{P}$ greatest when the value of the proportional gain is at its highest value.

This is one of the challenges of using a PI controller for a system. The issue can be solved by adding a derivative controller to the PI controller, thus making a PID controller. However, the speed of the PID controller may not be sufficient to generate the control signal for SoC. Hence, the best method is to use the optimum value of the proportional gain to dampen the overshooting and perform the power sharing and energy balancing at the optimum speed (in the case study, an optimal value for $K_{p b}$ would be 250). 


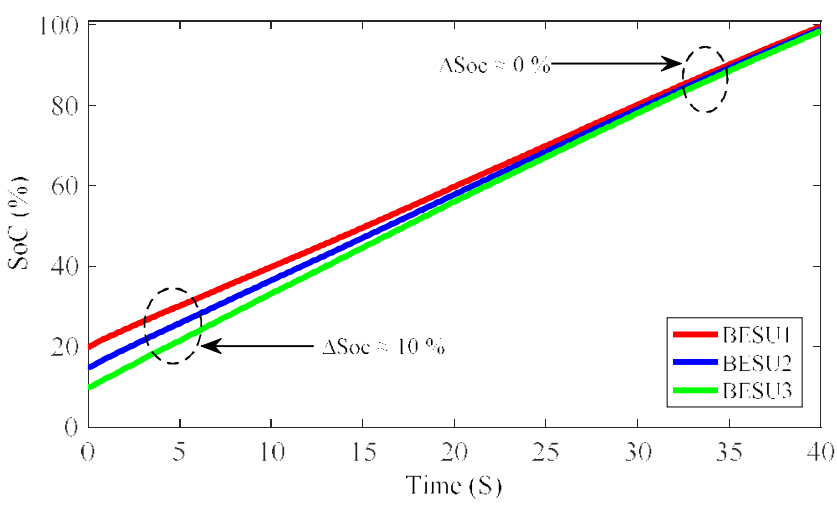

Fig.6 Energy balancing in charging period

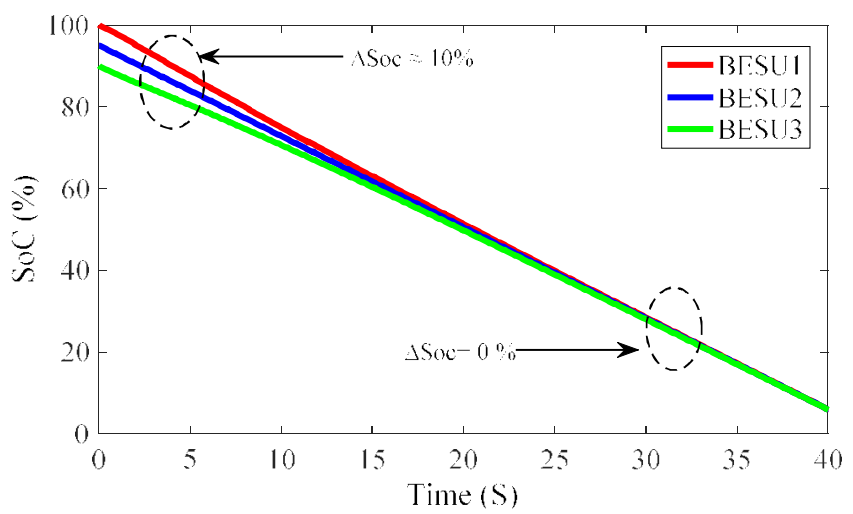

Fig.7 Energy balancing in discharging period

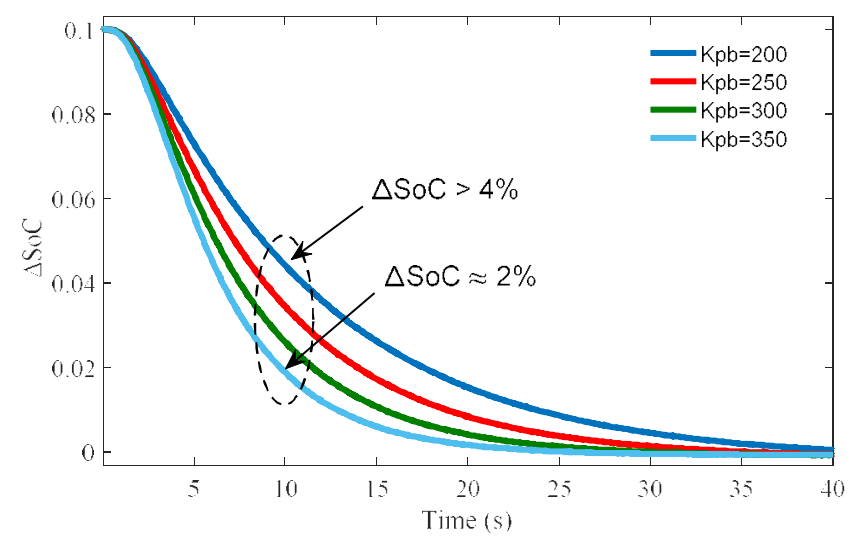

Fig. 8 Effects of the proportional gain $\left(K_{p b}\right)$ on the speed of energy balancing

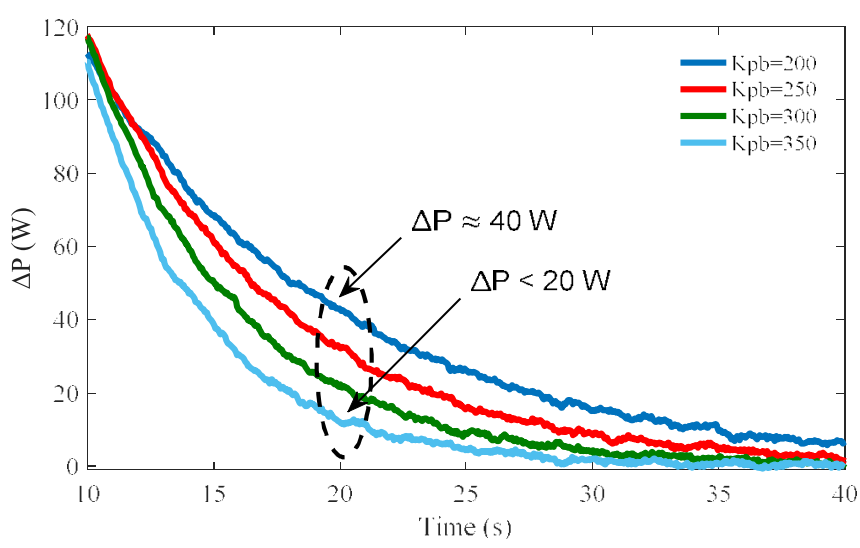

Fig.9 Effects of the proportional gain $\left(K_{p b}\right)$ on equalization of power

\section{CONCLUSION}

The main challenge of employing a battery energy storage system in the modern grid is controlling the system, especially given a distributed format. Equalization of stored energy in different units then presents a second challenge in such systems. Thus, a hierarchical control consisting of modified droop control (primary control), and a distributed control strategy for generating an accurate control signal for SoC (secondary control), are discussed in this paper. Based on this research, the conventional droop control needs to be modified in order to provide the optimum power sharing, leading to the equalization of the stored energy in different battery storage units. A PI controller is used for the upper control level (secondary control) and the value of the proportional gain is the most effective factor for increasing the speed of energy convergence. Future work in this area should investigate and evaluate the control method when one or several units are lost simultaneously. Moreover, optimizing the communication between different units is another open research question in such systems.

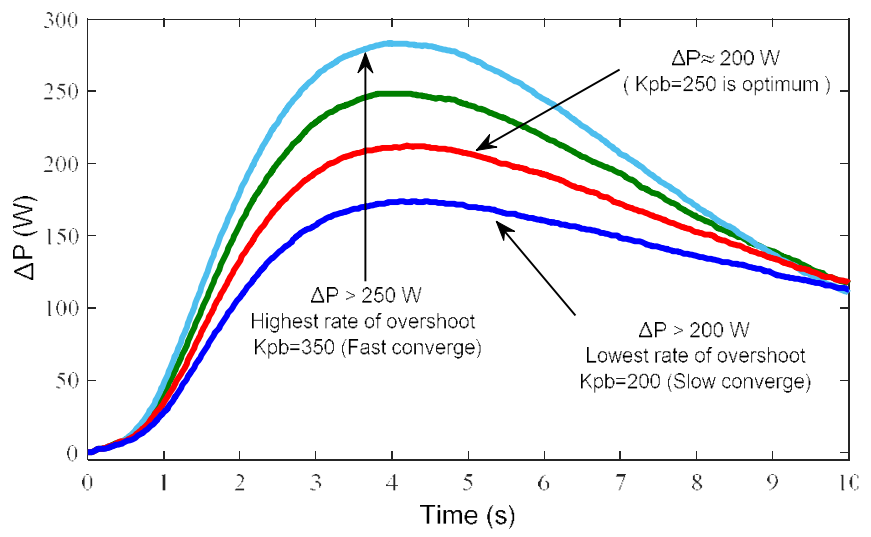

Fig.10 Effects of the proportional gain $\left(K_{p b}\right)$ on the rate of overshoot in preliminary output power

\section{References}

[1] J. M. Guerrero, J. C. Vasquez, J. Matas, L. G. De Vicuña, and M. Castilla, "Hierarchical control of droop-controlled $\mathrm{AC}$ and DC microgrids - A general approach toward standardization," IEEE Trans. Ind. Electron., 2011.

[2] O. Palizban and K. Kauhaniemi, "Power sharing for distributed energy storage systems in AC microgrid: Based on state-of-charge," in Asia-Pacific Power and Energy Engineering Conference, APPEEC, 2016, vol. 2016-Janua.

[3] B. M. T. Lawder et al., "System ( BESS ) and Battery Management System ( BMS ) for Grid-Scale Applications," Proc. IEEE, vol. 102, no. 6, 2014.

[4] E. Reihani, S. Sepasi, L. R. Roose, and M. Matsuura, "Electrical Power and Energy Systems Energy management at the distribution grid using a Battery Energy Storage System ( BESS )," Int. J. Electr. Power Energy Syst., vol. 77, pp. 337-344, 2016.

[5] T. L. Vandoorn, J. D. M. De Kooning, B. Meersman, and L. Vandevelde, "Review of primary control strategies for islanded microgrids with power-electronic interfaces," Renew. Sustain. Energy Rev., vol. 19, pp. 613-628, 2013.

[6] J. M. Guerrero, S. Member, M. Chandorkar, and T. Lee, "Advanced Control Architectures for Intelligent 
Microgrids - Part I: Decentralized and Hierarchical Control," IEEE Trans. Ind. Electron., vol. 60, no. 4, pp. 1254-1262, 2013.

[7] O. Palizban and K. Kauhaniemi, "Secondary control in AC microgrids: Challenges and solutions," in SMARTGREENS 2015 - 4th International Conference on Smart Cities and Green ICT Systems, Proceedings, 2015.

[8] Q. Shafiee, J. M. Guerrero, and J. C. Vasquez, "Distributed secondary control for islanded microgrids-a novel approach," IEEE Trans. Power Electron., vol. 29, no. 2, pp. 1018-1031, 2014.

[9] A. Bidram, S. Member, A. Davoudi, F. L. Lewis, and S. S. Ge, "Distributed Adaptive Voltage Control of InverterBased Microgrids," IEEE Trans. ENERGY Convers., vol. 29, no. 4, pp. 862-872, 2014.

[10] T. Morstyn, S. Member, B. Hredzak, and S. Member, "Control Strategies for Microgrids with Distributed Energy Storage Systems: An Overview," IEEE Trans. Smart Grid, vol. 3053, no. c, pp. 1-15, 2016.

[11] S. Jeon, J. Yun, and S. Bae, "Comparative Study on the Battery State-of-Charge Estimation Method," Indian J. Sci. Technol., vol. 8, no. October, pp. 1-6, 2015.

[12] C. A. Ooi, "Balancing Control for Grid-scale Battery Energy Storage Systems," Cardiff University, 2016.

[13] K. Worthmann, C. M. Kellett, S. Member, P. Braun, L. Grüne, and S. R. Weller, "Distributed and Decentralized Control of Residential Energy Systems Incorporating Battery Storage," IEEE Trans. Smart Grid, vol. 6, no. 4, pp. 1914-1923, 2015.

[14] T. R. Oliveira, W. Wodson, A. Gonçalves, and P. F. Donoso-garcia, "Distributed Secondary Level Control for Energy Storage Management in DC Microgrids," IEEE Trans. Smart Grid, vol. 8, no. 6, pp. 2597-2607, 2017.

[15] X. Lu et al., "Hierarchical Control of Parallel AC-DC Converter Interfaces for Hybrid Microgrids," IEEE Trans. Smart Grid, vol. 5, no. 2, pp. 683-692, 2014.

[16] O. Palizban and K. Kauhaniemi, "Hierarchical control structure in microgrids with distributed generation: Island and grid-connected mode," Renew. Sustain. Energy Rev., vol. 44, pp. 797-813, 2015.

[17] X. Lu et al., "State-of-Charge Balance Using Adaptive Droop Control for Distributed Energy Storage Systems in DC Microgrid Applications," IEEE Trans. Ind. Electron., vol. 61, no. 6, pp. 2804-2815, 2014.

[18] O. Palizban and K. Kauhaniemi, "Distributed cooperative control of battery energy storage system in AC microgrid applications," J. Energy Storage, vol. 3, 2015.

[19] T. Dragǐ, J. C. Vasquez, and D. Skrlec, "Supervisory Control of an Adaptive-Droop Regulated DC Microgrid With Battery Management Capability," IEEE Trans. POWER Electron., vol. 29, no. 2, pp. 695-706, 2014.

[20] T. Morstyn, S. Member, B. Hredzak, S. Member, and G. Vassilios, "Distributed Cooperative Control of Microgrid Storage," IEEE Trans. POWER Syst., vol. 30, no. 5, pp. 2780-2789, 2015. 